\title{
Moschata-Hybriden, die ersten öfter blühenden Strauchrosen
}

\author{
Eilike Vemmer
}

\begin{abstract}
The small rose group of Moschata hybrids (Hybrid Musk Roses) and its breeders are described. Most Moschata roses originated in the early 20th century from the German cultivar 'Trier'. Today most cultivars are from Belgium. Now most of them come from Belgium. They are just about to be known in Germany.

\section{Zusammenfassung}

Die kleine, aber bedeutende Strauchrosengruppe der Moschata-Hybriden wird mit ihren Züchtern und den wichtigsten Sorten vorgestellt. Die meisten Moschata-Hybriden sind zu Beginn des 20. Jahrhunderts aus der deutschen Züchtung 'Trier' in England entstanden. Viele von ihnen sind nun belgische Züchtungen, die in Deutschland erst allmählich bekannt werden.
\end{abstract}

\section{Was sind Moschata-Hybriden?}

Die bekannten englischen Gartenbuchautoren Roger Phillips und Martyn Rix äußern sich zu den Moschata-Hybriden (englisch Hybrid Musk Roses): „This is a small group, but it includes some of the very best of all garden roses." Man versteht unter Moschata-Hybriden eine kleine Gruppe öfter blühender Strauchrosen, meist duftend und mit elegant überhängendem Wuchs. Sie zeigen eine reiche Blütenfülle, haben meist kleine bis mittelgroße Blüten und blühen besonders stark im Herbst. Typisch ist, dass aus ihrer Basis den ganzen Sommer über immer wieder neue Blütentriebe wachsen, die im selben Jahr noch blühen. Sie sind näher mit Rosa multiflora als mit Rosa moschata verwandt und entstanden zu Beginn des 20. Jahrhunderts.

Obwohl in dieser Gruppe so wunderschöne, dankbare Gartenrosen zu finden sind, wurden sie von Anfang an wenig beachtet. Viele Rosenkäufer möchten eher große, gefüllte Blüten und schenken einer gefälligen Strauchform kaum Aufmerksamkeit. Auch die reiche Blüte bis in den Winter wird kaum beachtet.

\section{Entstehung und erste Züchter}

$\mathrm{Zu}$ Beginn des 20. Jahrhunderts entstand aus 'Aglaia', einer gelblichen Multiflora-Kletterrose, bei dem Trierer Züchter Peter Lambert 1904 eine öfter blühende Strauchrose mit kleinen, cremeweißen, duftenden Blüten mit goldenen Staubgefäßen, die auch als Kletterrose gepflanzt werden konnte. LAMBERT nannte sie nach seiner Heimatstadt 'Trier'. Diese Rose sah der eng- lische Rosenexperte Joseph H. Pemberton und schrieb, dies sei die erste Rose einer neuen Klasse und werde bald in allen Gärten stehen. Pemberton begann gemeinsam mit seiner Schwester Florence aus der Rose 'Trier' neue Strauchrosen zu züchten und nannte sie wegen des Duftes und der Herbstblüte 'Hybrid Musk Roses'.

Es sind keine Hybriden der Rosa moschata, obwohl diese in deren Ahnenreihe vorkommt. Der englische Rosenspezialist Norman Young meinte, dass selbst die ersten von Pembertons Hybriden acht Generationen von $R$. moschata entfernt waren. Er bezweifelt, ob überhaupt noch irgendeine Charakteristik dieser Art in auch nur einer von ihnen gefunden werden kann.

Einige dieser Pemberton-Rosen sind bis heute beliebt wie die weiße 'Moonlight' (1922), die cremefarbene 'Penelope' (1924), 'Cornelia' (1925) mit mehrfarbigen Blüten, die stark duftende, zartrosa 'Felicia' (1928) und die rote, für die Züchtung wichtige 'Robin Hood' (1927).

\section{Weitere Züchter und ihre Rosen}

Nachfolger der Geschwister Pemberton wurden ihr Gärtner John Bentall und besonders dessen Frau AnN. Ihre bekanntesten Rosen sind neben der weit verbreiteten Polyantharose 'The Fairy' die weißrosa 'Ballerina' (1937) und die gelbe 'Buff Beauty' (1939).

Auch Peter Lambert züchtete mit seiner 'Trier' weiter. Viel Charme hat seine 'Heideröslein' (1932) mit mittelgroßen, zartrosa Blüten- 
schalen. Leider sind die meisten MoschataHybriden von Peter Lambert, die auch 'Lambertiana' genannt werden, nur noch in wenigen Spezialsammlungen zu finden.

Wilhelm Kordes II. züchtete aus 'Robin Hood' Anfang der 1930er Jahre zwei Strauchrosen, 'Wilhelm' und 'Eva'. Letztere wurde Ausgangssorte anderer Strauchrosen wie 'Erfurt', 'München', 'Sangerhausen'. Auch sein Sohn Reimer Kordes benutzte 'Robin Hood' zur Züchtung seiner wohl berühmtesten Beet- oder Strauchrose 'Schneewittchen'. Aus 'Robin Hood' züchtete LAMBERT seine berühmte 'Mozart' (1937). Diese sieht wie der große, dunklere Bruder zu der zierlichen 'Ballerina' aus. 'Robin Hood' wurde auch von dem Niederländer DE Ruiter zur Zucht seiner Compacta-Zwergrosen benutzt ('Alberich', 'Degenhardt') und häufig von dem belgischen Züchter Louis Lens z. B. für die Strauchrosen 'Pink' und 'Red Robin'.

Andere Züchter in Europa gingen ähnliche Wege, um öfter blühende Strauchrosen zu erzielen. JACK HARKNess schuf aus 'Ballerina' die 'Red Yesterday' oder 'Red Ballerina' oder 'Marjorie Fair', wie sie in England heißt. PemberTONs 'Vanity' wurde ein Elternteil der Austinrose 'Scintillation', 'Ballerina' ein Elternteil von 'Francine Austin'.

\section{Louis Lens, der wichtigste Züchter der Moschata-Hybriden}

Die meisten Moschata-Hybriden stammen von Louis Lens aus Belgien und heute von seiner Nachfolgerin Ann Velle-Boudolf (LensRoses). Lens hat von etwa 1980 bis zu seinem Tode 2001 über 50 Moschata-Hybriden geschaffen, die zwar in England und Frankreich beliebt sind, in Deutschland aber kaum bekannt wurden. LENs benutzte häufig 'Ballerina' und 'Trier' zur Zucht. Viele Moschata-Hybriden von Lens und Velle-Boudolf erhielten internationale Auszeichnungen. 2012 bekam 'Guirlande d'Amour' in Deutschland sogar das ADR-Prädikat. Die ADR-Prüfung (Allgemeine

Abb. 1 (oben): 'Ballerina'.

Abb. 2 (unten): 'Schengen-Rose'.
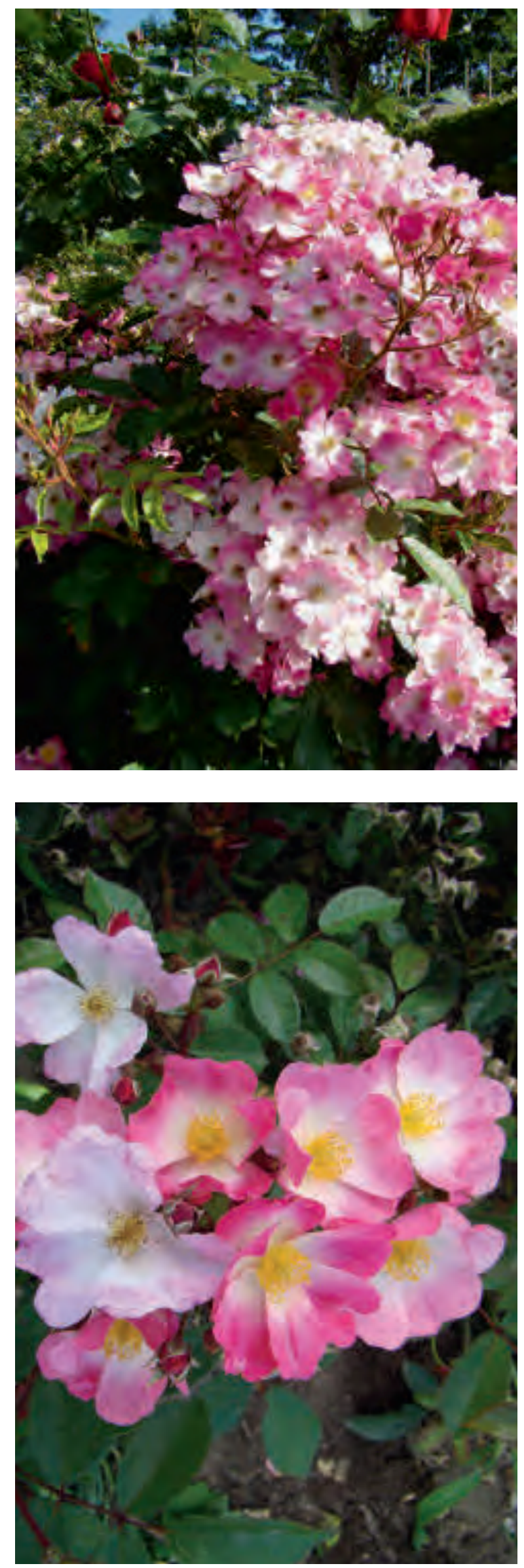

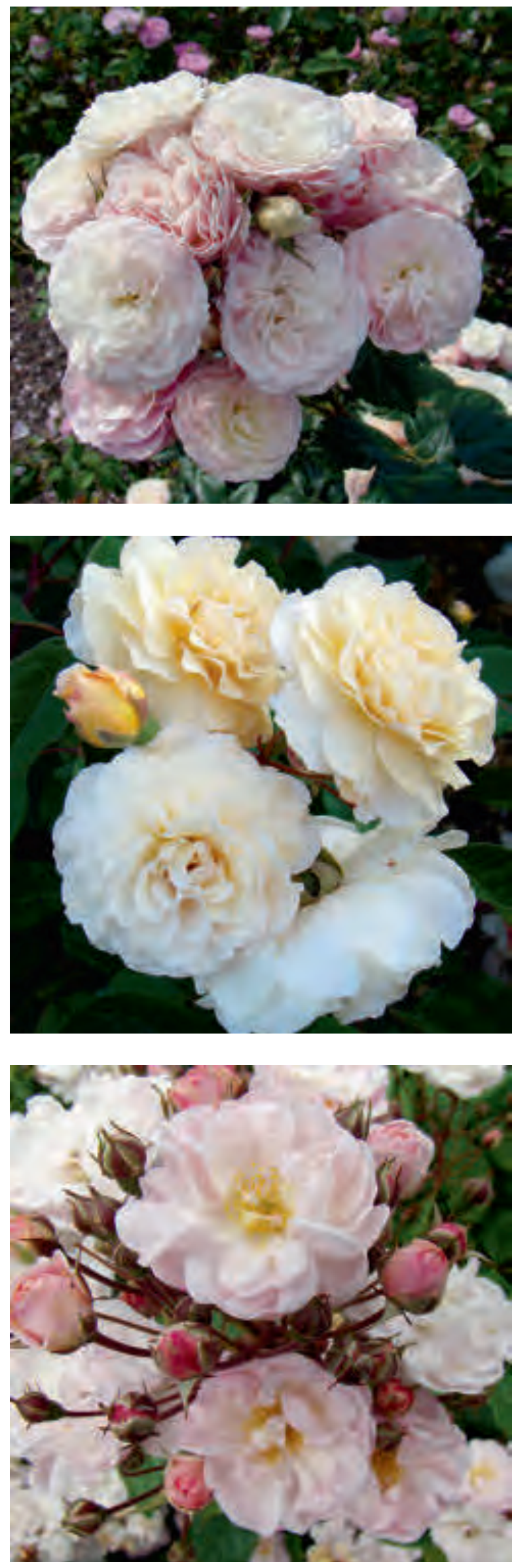

Deutsche Rosenneuheiten-Prüfung) gilt weltweit als strengste Auslese gesunder, blühreicher Rosen. Zur Zeit steht die Lens-Rose 'Hedi Grimm' in der ADR-Prüfung.

Anknüpfend an 'Mozart' schuf Louis Lens eine ganze Musikerserie in Rosa, Rot und Violett wie 'Paganini' (1989), 'Puccini' (1984), 'Ravel' (1988), 'Schubert' (1984), 'Sibelius' (1984), 'Verdi' (1984).

Eine Spitzensorte ist sicherlich die weiße, duftende 'Guirlande d'Amour' (1993), die man als Strauch- oder Kletterrose pflanzen kann. Auch andere weiße Lens-Moschata-Hybriden lohnen sich sehr im Garten wie 'Hedi Grimm' (2005); die Expertin für historische Rosen schuf die Rosensammlung im Park Kassel-Wilhelmshöhe, 'Waterloo' (1996), ein belgischer Ort berühmt durch die Schlacht gegen NAPOLÉON 1815, 'Schoone Gezelle Blomme' (1999), ein gesunder, winterharter Dauerblüher zu Ehren eines flämischen Dichters benannt, 'Jacqueline Humery' (1995), eine französische Gartenjournalistin.

Etwas Besonderes ist seine Kreuzungsserie 'Trier' x Rosa chinensis 'Mutabilis', aus der 'Plaisanterie' (1996) die bekannteste und wohl auch beste ist. Es handelt sich um einen bunten, duftenden Strauch mit Blüten von Mai bis zum Frost. 'Plaisanterie' kann auch als kleine Kletterrose gepflanzt werden. Aber auch die gefüllte 'Apricot Bells' (1999) ist zu empfehlen oder die rotlaubige 'Souvenir de Louis Lens' (2011).

Sehr gute, gesunde und schöne LensMoschata-Hybriden sind z. B. die rosarote 'Françoise Drion’ (1995) mit langen, pyramidalen Blütenständen, 'Heavenly Pink' (1997), ihre reiche Blüte in „himmlischem" Rosa bezaubert besonders im Herbst, 'Gravin Michel d'Ursel' (1994), benannt nach der 2003 verstorbenen Rosenexpertin aufSchloss Hex. 'Rosalita' (1997) wurde von der belgischen Königin auf ihren

Abb. 3 (oben): 'Bouquet Parfait' aus der Nähe.

Abb. 4 (Mitte): 'Buff Beauty'.

Abb. 5 (unten): 'Jean Stephenne'.

Abb. 6 (Seite 29): 'Bouquet Parfait'. 


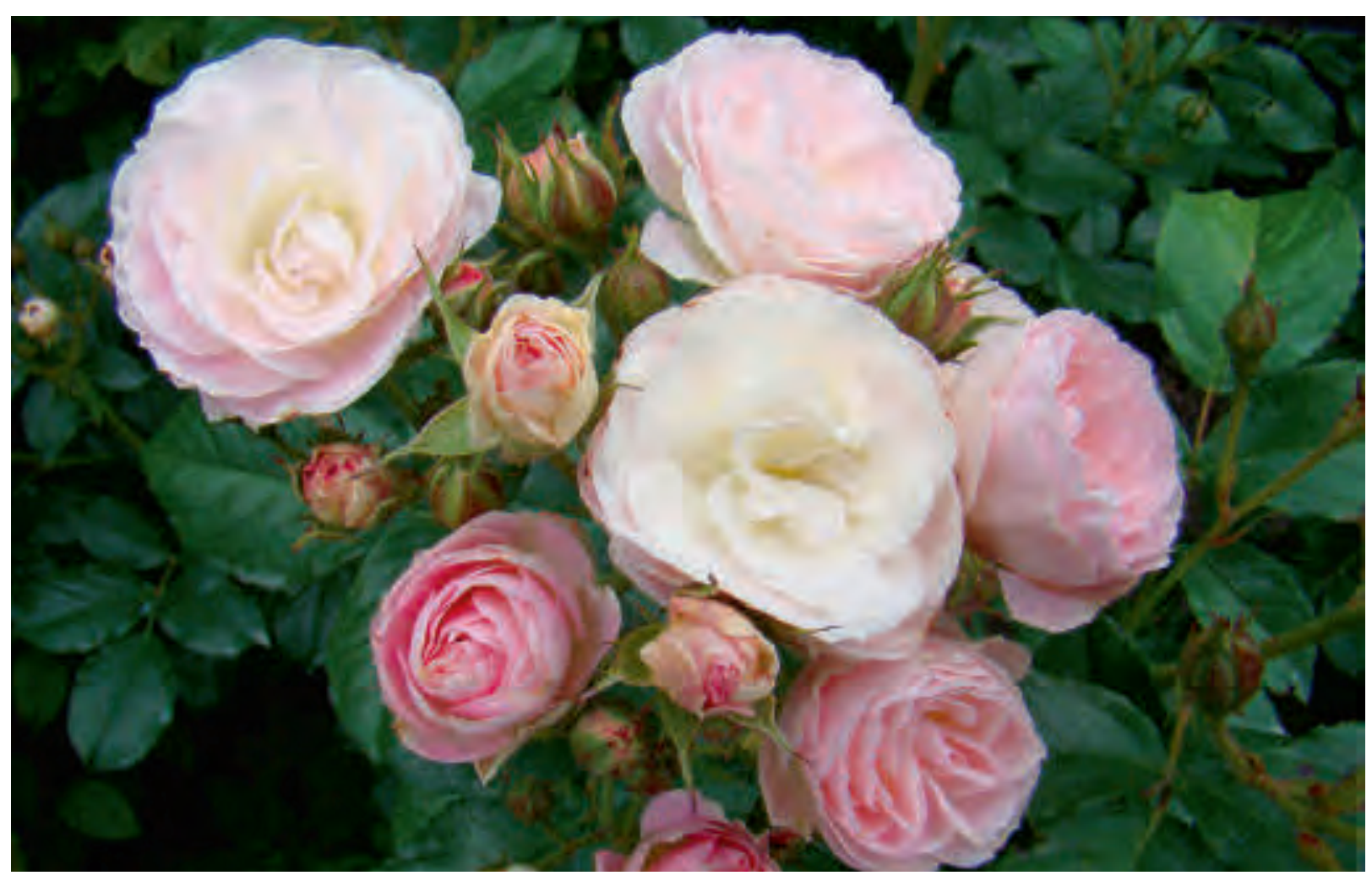

Kindernamen getauft. Bei 'Bouquet Parfait' (1989) ist monatelang wirklich jeder Blütenzweig ein vollkommenes Bukett.

\section{Ann Velle-Boudolf und ihre Rosen}

1992 übernahmen AnN und Rudy Velle die Rosenschule Lens. Ann Velle-Boudolf ist zur Zeit wohl die bedeutendste Moschata-Hybriden-Züchterin. Auch ihre Rosen bekamen schon viele Auszeichnungen (Velle Boudolf 2012).

Fuchsienrote Blüten hat 'Dinky' (2002), eine Rose für Regengebiete. 'Gaard um Titzebierg' (2005) wurde zu Ehren eines Luxemburger Rosengartens benannt, ein zartviolett-rosa Dauerblüher. Der Duft der zartrosa bis weißen 'Jean Stephenne' (2006) ist betörend, die Blattgesundheit und Winterhärte überragend, sie braucht allerdings etwas Platz und eigentlich für Deutschland einen zugkräftigeren Namen.

Auffallend große, rote Blüten mit weißem Herz hat 'Laurent Nicolas' (2009), ich sah sie von Juni bis November nie ohne Blüten. 2010 kamen zwei Moschata-Hybriden von ANN Velle-Boudolf in den Handel: die weiße 'Elisabeth Oberle' (in der Schweiz 'Kartause
Ittingen') mit gelben Knospen und Wildrosencharakter und die mehrfarbig rosa 'Schengen Rose' mit weißem Herz, ein schöner, kugeliger Strauch und reiche Blüte. Sie wurde zum 25-jährigen Bestehen des Schengen-Abkommens 2010 in Luxemburg getauft. Kugelige, gefüllte, gelbe Blüten hat 'La Feuillerie' (2011). Ann Velle-Boudolf hat eine glückliche Züchterhand (Vemmer 2011)!

\section{Eigenschaften der Moschata-Hybriden}

Die Blüte der Moschata-Hybriden ist im Herbst oft auffallender als im Sommer, wenn alle Rosen blühen. Bei uns in Norddeutschland frieren viele der Moschata-Hybriden in strengeren Wintern zurück, bauen sich aber sehr schnell wieder auf, weil sie den Sommer über ständig neue Blütentriebe aus der Basis hervorbringen. Alle blühen in mehr oder weniger großen Blütenständen, und viele haben einen angenehmen Duft (Brumme \& Vemmer 2010).

Die Vitalität der Moschata-Hybriden ist sehr unterschiedlich, abhängig von den eingekreuzten Rosen. Man kann weder sagen, alle Moschata-Hybriden sind gut oder widerstandsfähig, noch von einem bestimmten Züchter sind alle 

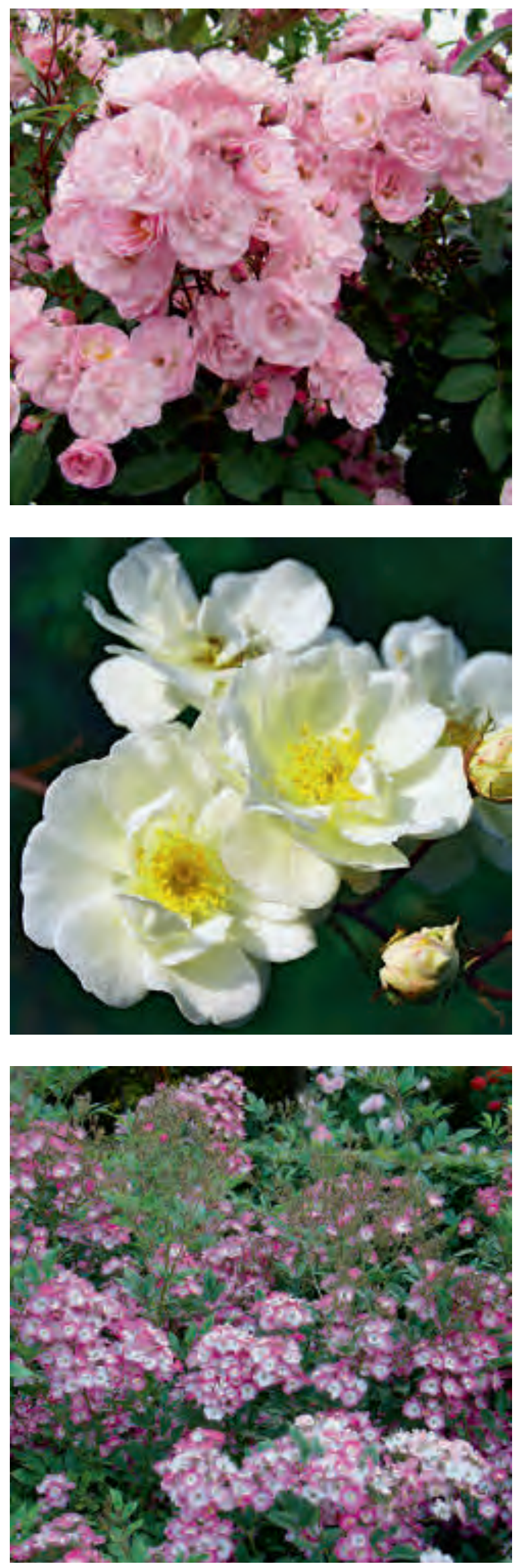

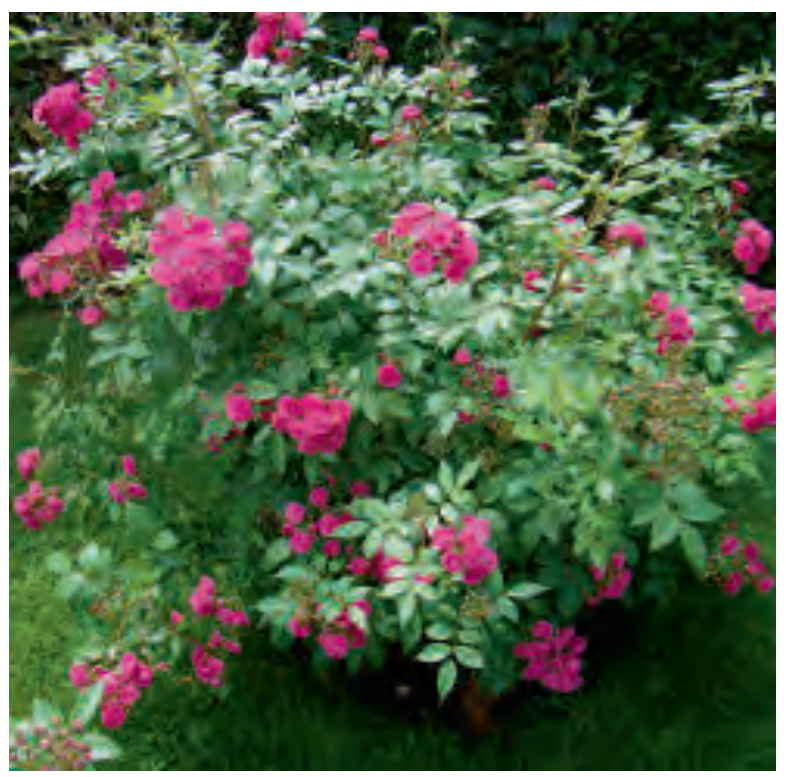

gesund. Genau das Gleiche gilt für die Frosthärte. Dazu muss man deutlich sagen, dass Gesundheit auch von Standort, Klima und der Pflege abhängt, nicht nur von der Sorte. Eindeutig sind die neueren Sorten von Louis Lens und Ann Velle-Boudolf an Gesundheit den Pemberton-Hybriden überlegen. Die im Herbst 2006 herausgekommene 'Jean Stephenne' von Velle-Boudolf ist eine der gesundesten Rosen, die ich kenne. Sie steht seit mehr als 10 Jahren in unserem Garten in der Nähe von Braunschweig und gedeiht dort prächtig.

Moschata-Hybriden sind wirklich charmante Rosen für jeden Garten!

\section{Literatur}

Velle-Boudolf. A. 2012: „Uitbundig bloeiende Rozen. De mooiste Moschata van Lens Roses." - Lannoo, Belgien. Brumme, H. \& Vemmer, E. 2010: Moschata-Hybriden. - Sangerhausen.

Vemmer, E. 2011: „Florence Pemberton, Ann Bentall, Ann Velle - Züchterinnen von Hybrid Musk Roses. 24. Kasseler Rundgespräch. - Dortmund.

\section{Internetseite}

www.lens-roses.com

Abb. 7 (oben links): 'Heavenly Pink'.

Abb. 8 (oben rechts): 'Dinky'.

Abb. 9 (Mitte): 'Moonlight'.

Abb. 10 (unten): 'Mozart'. 